\title{
Image-processing algorithms for behavior analysis of group-housed pigs
}

\author{
J. HU and H. XIN \\ Iowa State University, Ames, Iowa
}

\begin{abstract}
Computational algorithms of image processing were developed and evaluated to select, by motion detection, images of resting artificial pigs and to segment the pigs (mixture of black and white pigs) from their background. Motion detection of the pigs was implemented by detecting interframe differences of postural behavioral images. This algorithm combines the advantages of likelihood ratio method and shading model method and shows a stable performance under noisy and dynamic illumination conditions. Segmentation of the pigs from their background was implemented by employing multilevel thresholding and background reference techniques. The algorithm automatically determines the number of thresholds needed and produces satisfactory segmentation when both black and white pigs with different image intensities are present at the same time (the most complicated situation). The reference background image is updated so that temporal changes in illumination and/or spatial changes of the pen condition have little effect on the performance of image segmentation. The algorithm employs statistical models of the pigs and background and Bayes hypothesis testing to obtain and update the exposed portion of the reference background. Linear filters were used in this process for updating the parameters. These algorithms will serve as essential components for a novel, behavior-based, interactive approach to assess and control thermal comfort of group-housed pigs, which is expected to result in enhanced animal health and well-being.
\end{abstract}

The dependence of animal well-being and the resultant production performance on the thermal environment necessitate efficient environmental control. It is well known that animal thermal comfort is affected by not only air temperature but also other factors such as drafts, radiation, floor conditions, nutritional plane, and health state (Boon, 1981; Geers, Goedseels, \& Parduyus, 1986). However, the standard method for assessing and controlling swine housing environment has been based on air temperature. This method, though providing some degree of protection on the well-being of the animals, is not interactive with the animals because it fails to integrate all other influencing factors. Consequently, a seemingly ideal air temperature can be a mismatch to the animal's real environmental needs.

Animal behavior-based environmental control has been proposed to overcome the shortcomings of the conventional controllers. The rationale is that animals are the integrators to all the physical, nutritional, and physiological factors, and, therefore, they are the best sensors to indicate the adequacy of the environment. Some initial work was conducted by Wouters et al. (1990) and Geers et al. (1991). However, the specially designed temperature gradient pigpen and the dependency of animal occupation percentage on body size and age, as used or revealed by

Journal Paper No J-18154 of the Iowa Agriculture and Home Economics Experiment Station, Iowa State University, Project No. 3355. Funding for this study was provided in part by the Special Research Initiation Grant of Iowa State University and the lowa Pork Producers Association. Correspondence should be addressed to H. Xin, Agricultural and Biosystems Engineering Department, 203 Davidson Hall, Iowa State University, Ames, IA 5001 1-3080 (e-mail: hxin@iastate.edu). these researchers, present challenges for realizing sucl an automatic system on a commercial production scale Xin and Shao (1997) proposed a six-step, behavior-basec system to automatically assess and control swine therma comfort (Figure 1). A feasibility study by Shao, Xin, anc Harmon (1997) used artificial neural network (ANN analysis of swine postural behavior images to classify their thermal comfort state, and the results were promis ing. The authors then investigated the efficacy of variou: feature extraction methods for the ANN classificatior (Shao, Xin, \& Harmon, 1998). They further pointed ou the needs for automatic selection of the postural image: and automatic segmentation of the pigs from their back ground for subsequent ANN classification (Shao, 1997) Although numerous methods have been developed in the general area (Pal \& Pal, 1993; Sahoo, Soltani, Wong, \& Chan, 1988), automatic image segmentation remains ont of the most difficult tasks in image analysis because of it: application-specific nature.

The objective of this study was to develop image. processing algorithms for automatic selection of postura behavioral images by motion detection and segmentatior of young pigs in order to facilitate future implementatior of the proposed behavior-based assessment and contro of the animal thermal comfort.

\section{METHOD}

\section{Experimental Setting and Data Collection}

A commercial, orange-colored plastic slat floor for young pigs wa: used in a lab-scale setting of the imaging system. Artificial pigs (botk white and black colors) in resting position were made from enlargec color photographs taken from previous live animal trials. The pig: 


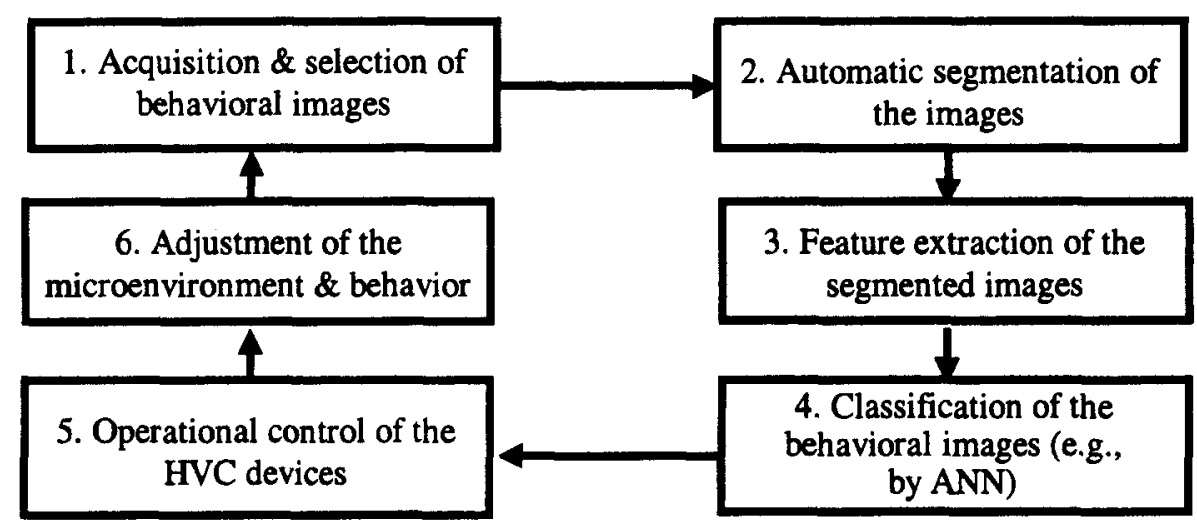

Figure 1. Block diagram of behavior-based assessment and control of swine thermal comfort (Xin \& Shao, 1997). HVC, heating, ventilation, and cooling.

were placed on the floor of the pigpen to form postural behavior patterns representing resting patterns under various (cold to hot) thermal conditions. A centrally located CCD camera overseeing the pigpen was used along with frame grabber and software (Matrox Electronic Systems Ltd., Dorval, Canada) installed in a PC to capture the experimental images. Illumination level was constant for a given set of captured images, including the background reference images and the behavioral images. All images were digitized into 256 intensity levels. Hu (1998) gave a more detailed description of the system setup.

Computer-generated image sequences of objects satisfying certain statistical properties (normally distributed with certain mean and variance) were also used to develop and evaluate the algorithms. System noise in this application was assumed to be additive and normally distributed with zero mean.

\section{Image Motion Detection}

Motion detection was used to select postural images of the resting pigs. This is needed because pigs in motion would generally not reflect the thermal conditions. Besides changes due to the pigs' movement, differences between images may also result from changes in illumination and/or noise during the digitizing process. Differences caused solely by feeding and manure dropping during the short time of capturing image sequences are much smaller than those caused by movement and illumination change and can be handled by selecting proper threshold. The objective was thus to find the motion detection method that would be robust to noise and illumination changes and could produce a reliable binary result: static or moving.

Likelihood ratio method. Several methods of using a block of adjacent pixels to reduce the sensitivity to noise have been developed. The commonly used statistics are the mean and variance of individual blocks. Yakimovsky (1976) used a likelihood ratio to determine whether two neighboring regions in the same image can be merged. It has been generalized for comparing regions of two consecutive frames of the same scene in an image sequence to detect motion. This likelihood $L(i)$ is calculated as:

$$
L(i)=\frac{\left[\left(\sigma_{i 1}^{2}-\sigma_{i 2}^{2}\right) / 2+\left(\left(\mu_{i 1}-\mu_{i 2}\right) / 2\right)^{2}\right]^{2}}{\sigma_{i 1}^{2} \sigma_{i 2}^{2}},
$$

where $\mu_{i 1}$ and $\sigma_{i 1}^{2}$ are the mean and variance of region $i$ in frame 1 ; $\mu_{i 2}$ and $\sigma_{i 2}^{2}$ are the mean and variance of region $i$ in frame 2 . If the likelihood ratio is less than a given threshold, these two areas are considered to come from the same intensity distribution, and, thus, no object motion has occurred.

Shading model method. Shading models are used for computer graphics. Skifstad and Jain (1989) used the shading model method to improve the performance of motion detection under changing illumination. This improvement stems directly from its definition of intensity $f(x, y)$ as the product of illumination $I$ and a pixel-based shading coefficient $S(x, y)$; that is,

$$
\begin{aligned}
& f(x, y)=I * S(x, y) \\
& S(x, y)=C_{p} *[\cos (i) *(1-d)+d]+W(i) *[\cos (s)]^{n},
\end{aligned}
$$

where $C_{p}$ is the reflection coefficient of the object at point $P$ for a certain wavelength; $i$ is the incident angle; $d$ is the environmental diffuse reflection coefficient; $W(i)$ is a function that gives the ratio of the specular reflected light to the incident light as a function of the incident angle; $s$ is the angle between the direction of the reflected light and the line of sight; and $n$ is a power that models the specular reflected light for each material.

Shading coefficient $S(x, y)$ is only related to the physical surface structure of the objects and the reflection properties of the surface material and is independent of illumination. Motion detection method based on this concept should thus be insensitive to illumination variance. Because it is difficult to obtain $S(x, y)$ without prior knowledge of the objects in scene, the intensity ratio of the same regions of the two frames is used:

$$
\frac{f_{1}(x, y)}{f_{2}(x, y)}=\frac{I_{1} * S_{1}(x, y)}{I_{2} * S_{2}(x, y)}
$$

If there is no object motion and thus no change of the associated physical structure, then

$$
\begin{aligned}
S_{1}(x, y) & =S_{2}(x, y) \\
\frac{f_{1}(x, y)}{f_{2}(x, y)} & =\frac{I_{1}}{I_{2}} .
\end{aligned}
$$

In any block of the image, all the intensity ratios between the pairs of pixels at the same location of two successive frames either equal unity if illumination is constant or equal some constant number if illumination changes. Under both situations, however, the variance of the ratio for that region equals zero. If there do exist motions of the objects in the scene, regardless of whether or not illumination changes, the variance of that ratio will be larger than zero because of the resultant changes of physical structure due to motion. Therefore, a nonzero variance indicates object motion, and it is insensitive to illumination change in this model.

In applying this method, successive frames need to be divided into blocks, and the variance of the intensity ratios of pixel pairs within each block is calculated. Thresholding will then classify each block into motion or still group. 


\section{Image Segmentation}

The final output of the image segmentation in this application was a binary image with the pigs in white pixels and the background in black pixels. This was done by automatic multilevel optimal thresholding. For every level $i$, starting from 1 to a maximum number of thresholds $C$ estimated beforehand on the basis of the application, an optimal threshold vector $T=\left[t_{1}, t_{2}, \ldots, t_{\mathrm{i}}\right]$ was obtained automatically that maximizes an optimality measure $O(T)$. Among the obtained threshold vectors for different levels, a homogeneity measure $U(T)$ was used to automatically select the vector that maximizes the homogeneity as the final output. Because of the use of difference image and extended histogram (described below), thresholds located on the nearest right side and left side of zero were used to remove the background. The steps are listed below.

Background reference image formation. Five floor images were successively captured, and an average image was computed to represent the initial pig-free background condition. On the basis of this arrangement, it is reasonable to treat the reference image as a noise-free image whose pixels have the mean intensity values of the individual images at the corresponding pixel locations, assuming that the intensity value at every pixel is a random variable.

Difference image and extended histogram. The difference image was formed by subtracting the reference image from a pigladen image. If the illumination condition has not changed noticeably since capturing of the reference image, the differences between the pairs of the corresponding background pixels will be around zero. Hence, significant difference values would arise from subtraction between the object pixels and the background pixels. Although the specific threshold values cannot be easily obtained, the ranges of those values can be known. The subtraction also eliminates the possible peaks in the histogram stemming from the background regions. An extended histogram can be obtained by extending the intensity level range to 512 (i.e., from -256 to 255 ). The extended histogram allows separation of the positive and negative values in the difference image. This arrangement makes it more meaningful to employ the multilevel thresholding technique because optimal thresholds can always be computed independent of the shape of the histogram.

Multilevel optimal thresholding. An iterative multilevel thresholding technique was proposed by Yin and Chen (1997) for finding optimal multilevel thresholds to greatly reduce computational complexity of the direct extension of the optimal bi-level thresholding method to the multilevel case, which is usually an exhaustive search process. This multilevel optimal thresholding algorithm is illustrated by the flow chart shown in Figure 2 . The final output from the above algorithm is an optimal threshold vector $T=\left[t_{1}, t_{2}, \ldots, t_{c}\right]$ whose elements are the optimal thresholds. Two measures (i.e., optimality and homogeneity) are employed to guide the selection of the final result.

Optimality measure. Several optimal measures have been proposed for obtaining optimal global threshold (Lee, Chung, \& Park, 1990). However, not all of them are suitable for multilevel cases and iterative procedure. In the present study, optimal measures from Kapur, Sahoo, and Wong (1985) and Otsu (1979) were tested. The following are functional expressions for the bi-level and multilevel optimal measures.

Functions for bi-level optimal measures:

Kapur: $O(T)=H_{0}+H_{1}$

$$
\left\{\begin{array} { l } 
{ H _ { 0 } = - \sum _ { i = 0 } ^ { t - 1 } \frac { P _ { i } } { w _ { 0 } } \operatorname { l n } \frac { P _ { i } } { w _ { 0 } } } \\
{ H _ { 1 } = - \sum _ { i = t } ^ { L - 1 } \frac { P _ { i } } { w _ { 1 } } \operatorname { l n } \frac { P _ { i } } { w _ { 1 } } }
\end{array} \left\{\begin{array}{l}
w_{0}=-\sum_{i=0}^{t-1} P_{i} \\
w_{1}=-\sum_{i=t}^{L-1} P_{i}
\end{array}\right.\right.
$$

Otsu: $O(T)=w_{0} * w_{1} *\left(\mu_{0}-\mu_{1}\right)^{2}$

$$
\left\{\begin{array} { l } 
{ \mu _ { 0 } = - \sum _ { i = 0 } ^ { t - 1 } i * \frac { P _ { i } } { w _ { 0 } } } \\
{ \mu _ { 1 } = - \sum _ { i = t } ^ { L - 1 } i * \frac { P _ { i } } { w _ { 1 } } }
\end{array} \left\{\begin{array}{l}
w_{0}=-\sum_{i=0}^{t-1} P_{i} \\
w_{1}=-\sum_{i=t}^{L-1} P_{i}
\end{array}\right.\right.
$$

Functions for multilevel optimal measures:

Kapur:

$$
\left\{\begin{array}{l}
O(T)=\sum_{k=0}^{C} H_{k} \\
H_{k}=\sum_{i=t_{k}}^{t_{k+1}} \frac{P_{i}}{w_{k}} \ln \frac{P_{i}}{w_{k}} \\
w_{k}=\sum_{i=t_{k}}^{t_{k+1}-1} P_{i}
\end{array}\right.
$$

Otsu:

$$
\left\{\begin{array}{l}
O(T)=\sum_{k=0}^{C} w_{k} * w_{k+1} *\left(\mu_{k}-\mu_{k+1}\right)^{2} \\
\mu_{k}=\sum_{i=t_{k}}^{t_{k+1}-1} \frac{P_{i}}{w_{k}} \\
w_{k}=\sum_{i=t_{k}}^{i_{k+1}-1} P_{i}
\end{array}\right.
$$

where $i$ is the intensity level in the histogram, $P_{i}$ is the number of occurrence of level $i$ in the image, $L$ is the maximum intensity level of the image ( 256 for 8-bit image), $C$ is the maximum number of thresholds inclusive of the final optimum thresholds (derived from inspecting the histogram), $K$ is the current number of thresholds for determination of the optimum thresholds, and $t_{0}$ to $t_{k+1}$ are the current thresholds where $t_{0}=0$ and $t_{k+1}=L-1$. Optimum thresholds are those that correspond to the maximum optimal measure values.

Homogeneity measure. The commonly used uniformity measure was originally proposed by Levine and Nazif(1985), and it was based on the fact that uniformity of a feature over a region is inversely proportional to the variance of the values of the feature evaluated at every pixel belonging to that region. It has the form of

$$
U_{\partial}=1-2 * C * \sum_{j=0}^{C} \sum_{i \in R_{j}} \frac{\left(f_{i}-\bar{f}_{j}\right)^{2}}{\left[A_{\partial} *\left(f_{\max }-f_{\min }\right)^{2}\right]},
$$

where $\partial=$ image range under consideration;

$A_{\partial}=$ area of $\partial$ measured in the number of pixels;

$R_{j}=(j=1,2, \ldots, C)$, regions in $\partial$;

$C=$ total number of regions;

$f_{i}=$ feature value of pixel $i$ belonging to region $R_{j}$;

$\bar{f}_{j}=$ mean feature value within region $R_{j}$;

$f_{\min }=\min _{i \in d} f_{i}$

$f_{\max }=\max _{i \in \partial} f_{i}$.

\section{Reference Image Updating}

Updating of the reference image is needed because changes in the background conditions are generally inevitable. These changes may include color fading of the floor or surrounding walls, variation in illumination, shadows of the objects, pig manure on the floor, and so on. The algorithm developed in this study is based on statistical hypothesis testing. It aims to exclude the pixels that are influenced by the presence of pigs and to update the exposed portion of the background reference image - a process that minimizes distortion to the reference image. 


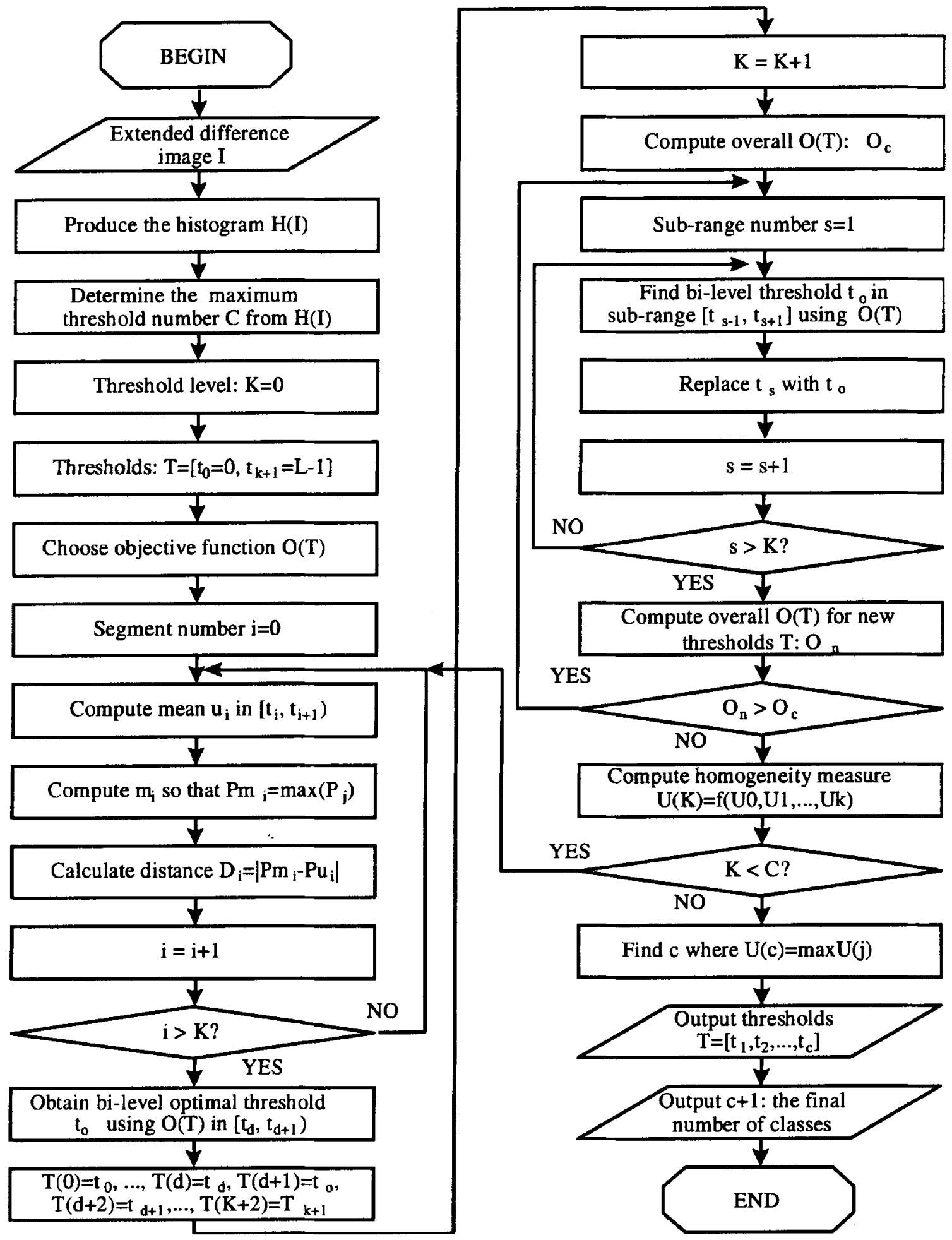

Figure 2. A flowchart illustration of multilevel thresholding based on the algorithm by Yin and Chen (1997).

Assumptions. Noise signals at different pixel positions are additive, independent, and identically distributed normal random variables with zero mean and variance $\sigma_{n}^{2}$. Intensity values of pixels within the image are random variables independent of one another. Reference image is a noise-free image, and the intensity value at every pixel in this image is the mean value of the intensity variable at that position. Thus, the intensity distribution of a captured background image $I$ at pixel $(x, y)$ can be expressed as a normal distribution. Intuitively, the variance of this normal distribution can be initially estimated by computing the intensity variance in the difference 
image between two background images. Intensity values at pixel locations belonging to objects (white or black pigs) are also assumed to have normal distributions.

Models. If the pixel at $(x, y)$ in the captured image belongs to the background, it is treated as the background event. If it belongs to the object, it is treated as the object event. The intensity distributions $F(x, y)$ can be modeled as

Background:

$F(x, y) \sim N\left[f_{r}(x, y), \sigma_{n}^{2}\right]=\frac{1}{\sqrt{2 \pi \sigma_{n}}} \exp \left\{-\frac{\left[f(x, y)-f_{r}(x, y)\right]^{2}}{2 * \sigma_{n}^{2}}\right\}$

Object: $F(x, y) \sim N\left(\mu_{o}, \sigma_{o}^{2}\right)$

$F(x, y) \sim \frac{1}{L}$,

where $f_{r}(x, y)$ is the mean intensity in the reference image at the same location as the pixel under examination, $\sigma_{n}^{2}$ is the variance of the noise signal, $\mu_{o}$ is the mean intensity of pixels belonging to the objects (white or black pigs in this case), and $\sigma_{o}^{2}$ is the corresponding variance. If normal distribution cannot be assumed under certain situations or the mean and variance cannot be estimated, the targeted object distribution could be modeled as uniform distribution, with $L$ being the maximum intensity level.

Hypothesis testing. When only black or white pigs are present in the scene, it can be handled as a binary hypothesis-testing problem. However, when both white and black pigs are present, threeway hypothesis testing would be a better model for the decision making.

For binary hypothesis testing, the hypotheses $H_{0}$ and $H_{1}$ corresponding to the background event and the object event under normal distribution and uniform distribution are

Normal Distribution:

Uniform Distribution:

$H_{0}: F(x, y) \sim N\left[f_{r}(x, y), \sigma_{n}^{2}\right]$

$H_{0}: F(x, y) \sim N\left[f_{r}(x, y), \sigma_{n}^{2}\right]$

$H_{1}: F(x, y) \sim N\left(\mu_{o}, \sigma_{o}^{2}\right)$

$H_{1}: F(x, y) \sim \frac{1}{L}$.

The decision of object versus background pixels will be made using the following Bayes rule, where $L$ is the likelihood ratio and $\tau$ is the threshold:

$$
\begin{aligned}
\delta[f(x, y)] & = \begin{cases}1 & L[f(x, y] \geq \tau \\
0 & L[f(x, y)]<\tau\end{cases} \\
L[f(x, y)] & =\frac{p_{1}[f(x, y)]}{p_{0}[f(x, y)]} \\
\tau & =\frac{\pi_{0}\left(C_{10}-C_{00}\right)}{\pi_{1}\left(C_{01}-C_{11}\right)} .
\end{aligned}
$$

$\pi_{j}$ represents the prior probability that hypothesis $j$ is true. In this application, if we assume that the possibility of pig location is completely random, $\pi_{1}$ can be estimated as the ratio of the area (number of pixels) occupied by pigs to that of the whole image. $\pi_{0}$ can be estimated as the ratio of the area of the exposed background to that of the whole image. $C_{i j}$ is the cost of accepting hypothesis $i$ when $j$ is actually true. Its value was chosen so that the largest cost was assigned to the most unwanted error. The absolute value of $C_{i j}$ is not important because of its ratio relationship with $\tau$ as long as all $C_{i j}$ are in the same range. They were selected in the range of 0 to 1 in our experiment.

When both white and black pigs are present in the scene, the hypothesis testing becomes a three-way test. In general, for $M$-way hypothesis testing, the hypotheses and decision rule become

$$
\begin{array}{lr}
H_{i}: F(x, y) \sim P_{i} & i=0,1, \cdots, M-1 \\
\delta[f(x, y)]=k & \text { if } \\
\sum_{j=0}^{M-1} \pi_{j} C_{k j} p_{j}[f(x, y)]=\min _{i} \sum_{j=0}^{M-1} \pi_{j} C_{i j} p_{j}[f(x, y)] .
\end{array}
$$

Updating reference image. The following weighted linear filter was used:

$$
f_{r}(x, y)=\lambda f_{r}(x, y)+(1-\lambda) f(x, y) \quad 0 \leq \lambda \leq 1 .
$$

This is a simple temporal, low-pass filter that recursively adjusts the intensity mean of every background pixel. $\lambda$ is the filter coefficient that weighs the relative importance of the original value and the newly captured value. Its value can be chosen on the basis of how fast the image scene changes. The slower the change, the larger the value of $\lambda$.

Assuming that the size of manure on the floor is much smaller than that of the pigs, a size threshold can thus be set to classify the pixels of manure into those of the background.

Estimation and updating of parameters. $\sigma_{n}^{2}$ can be estimated from the difference image between two background images. $\mu_{o}$ and $\sigma_{o}^{2}$ can be estimated originally from the segmented images of black or white pigs. On the basis of our observation and experimental results, it is reasonable to assume that the pixel intensity of the (white or black) pigs have a normal distribution with a mean of $\mu_{o}$ and a variance of $\sigma_{o}^{2}$ for each white- or black-colored pig. The estimated parameters of $\mu_{o}, \sigma_{o}^{2}$, and $\sigma_{n}^{2}$ can be updated on the basis of the segmentation result using the same technique for updating reference image. For example, $\sigma_{n}^{2}$ is updated using the following linear filter that acts on the exposed background regions:

$$
\sigma_{n}^{2}(k+1)=\partial \sigma_{n}^{2}(k)+(1-\partial) \sigma^{2} \quad 0 \leq \partial \leq 1 .
$$

$\pi_{j}$ is updated by calculating the area occupied by the pigs and the area of exposed background.

All the algorithms were originally implemented under UNIX system using DEC Alpha workstation of $300-\mathrm{MHz}$ speed by programming under the MATLAB 5.2 and Image Processing Toolbox 2.0 environment. They were later tested under MATLAB 5.2 environment on a Windows 95 Pentium 400 PC. Some images from the Image Processing Toolbox were used to test the algorithms. A graphic user interface (GUI) was developed to demonstrate the effectiveness of the algorithms. Snapshots of the GUI are shown in Figure 3 .

\section{RESULTS AND DISCUSSION}

\section{Image Motion Detection}

Block size influences motion detection results. The experiment showed that larger block sizes lead to lower misclassification rates. Selection of block size should be consistent with the intensity distribution of the pixels so that enough information is available within the block to accurately describe the distribution. This implies that block size is related to the size of the object under consideration and the distance between the camera and the object. The larger the object is in the scene, the higher percentage of the image will change due to motion of the object, and the larger the block size should be. In the case of normal distribution, an intensity distribution of large variance requires a large block size to minimize misclassification rate. In our experiment, this effect was apparent when block size was small. Once having reached a certain 

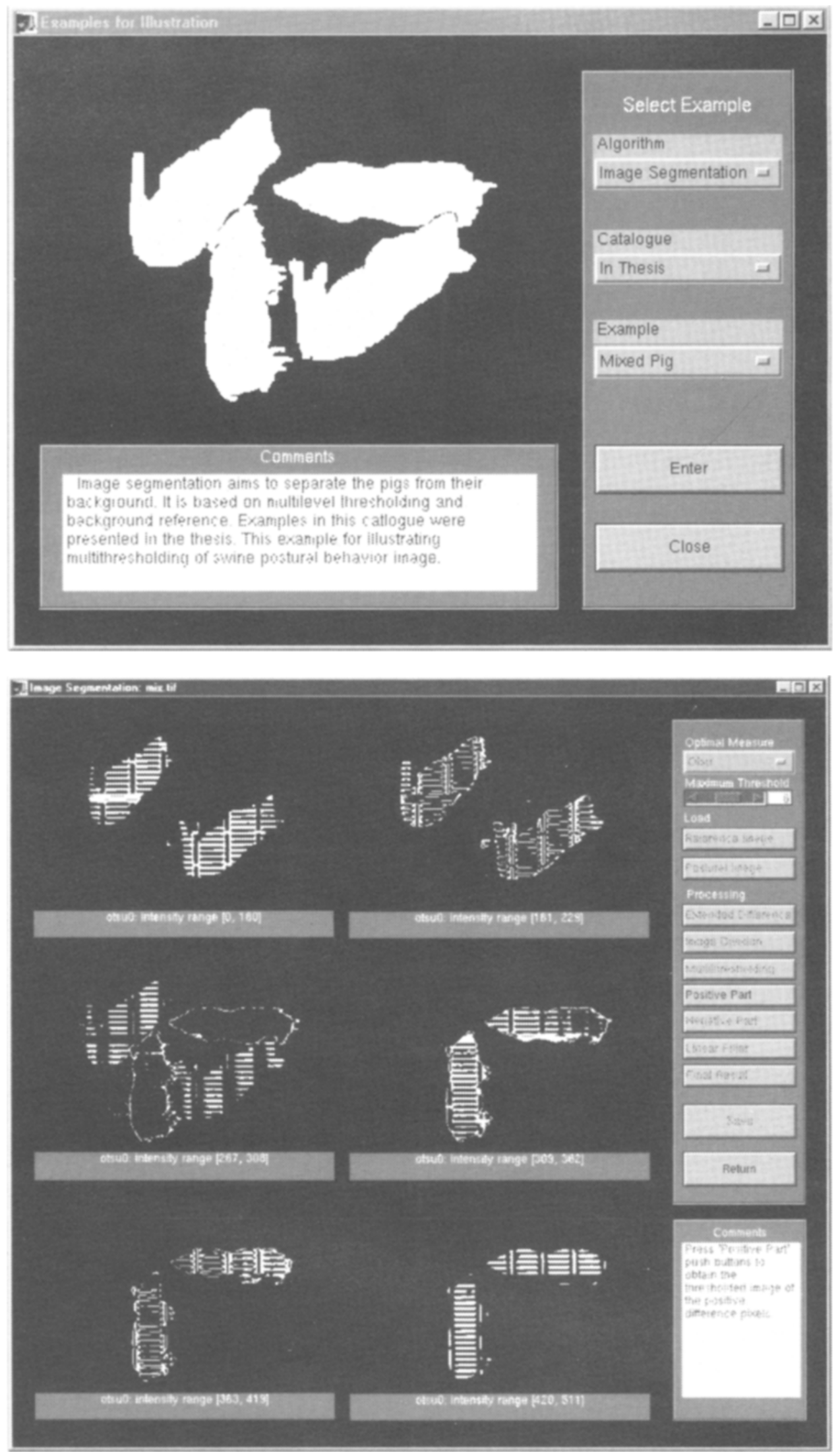

Figure 3. Snapshots of GUI used in the swine behavior image processing. 
level, further increase in block size will no longer produce improvement on classification rate.

However, further improvement could be obtained by inspecting motion detection results of the neighboring blocks of the block under examination. This is because if a motion has occurred in the scene as detected by one block, that motion would also affect the neighboring blocks. Thus, motion detection results of the neighboring blocks could be used to reduce potential errors caused by noise.

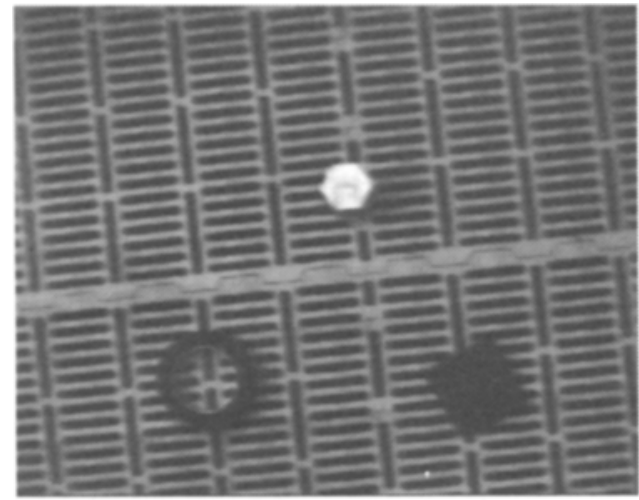

a) lower illumination

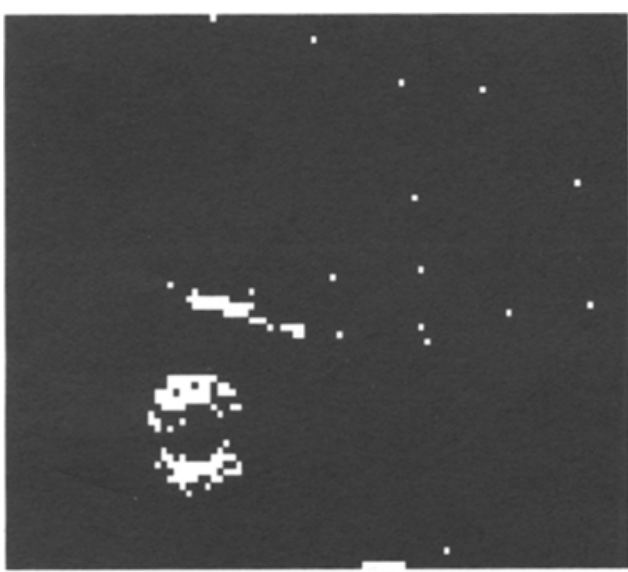

c) before intra-frame likelihood inspection

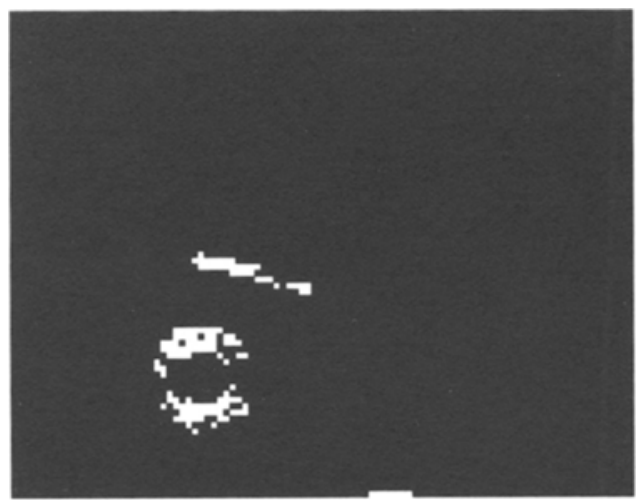

d) after intra-frame likelihood inspection

Shading model method $($ Threshold $=0.01)$

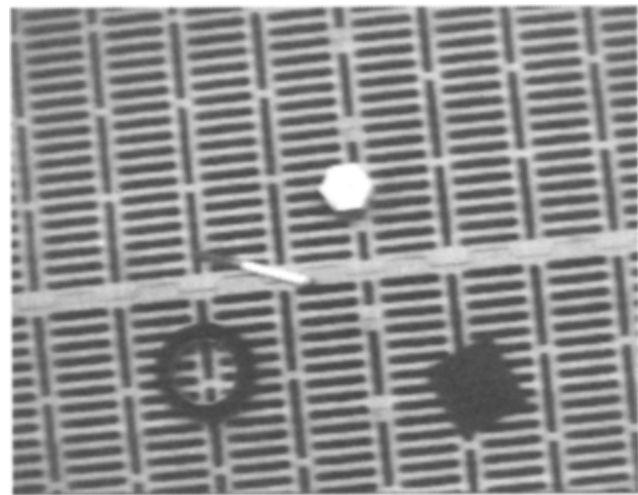

b) higher illumination

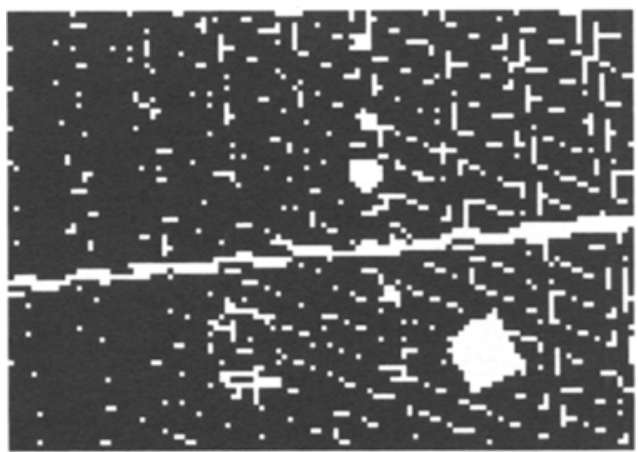

e) before intra-frame likelihood inspection

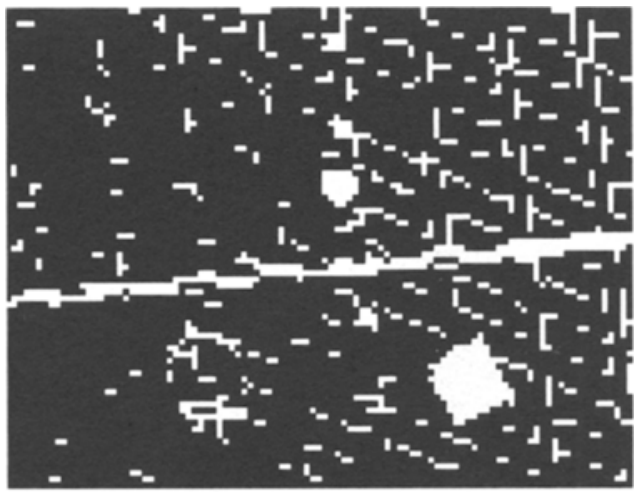

f) after intra-frame likelihood inspection

Likelihood model method (Threshold $=\mathbf{2 0}$ )

Figure 4. Advantage of the shading model method under changing illumination. 


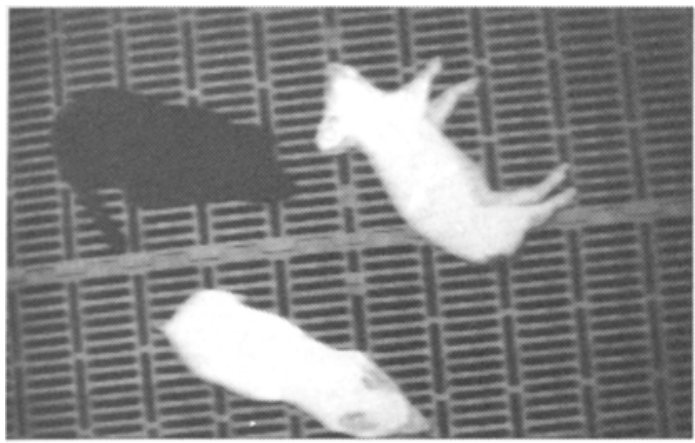

a) frame 1

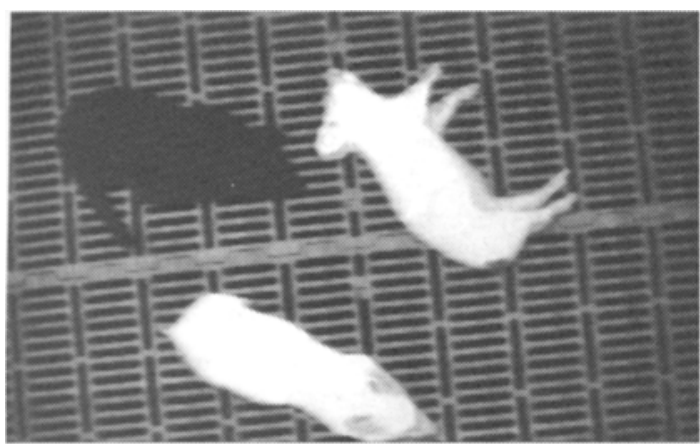

b) frame 2

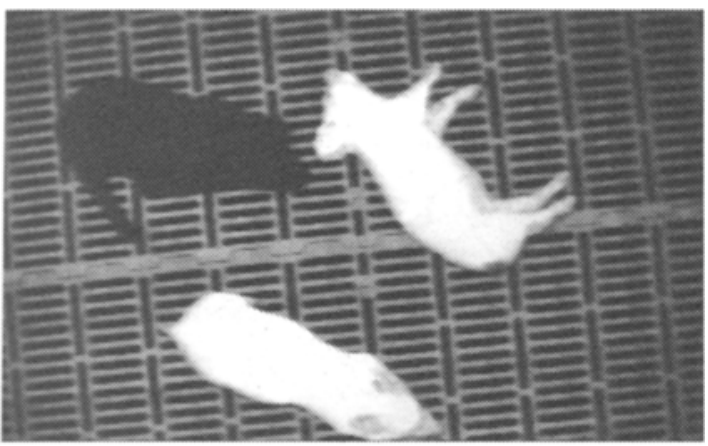

c) frame 3

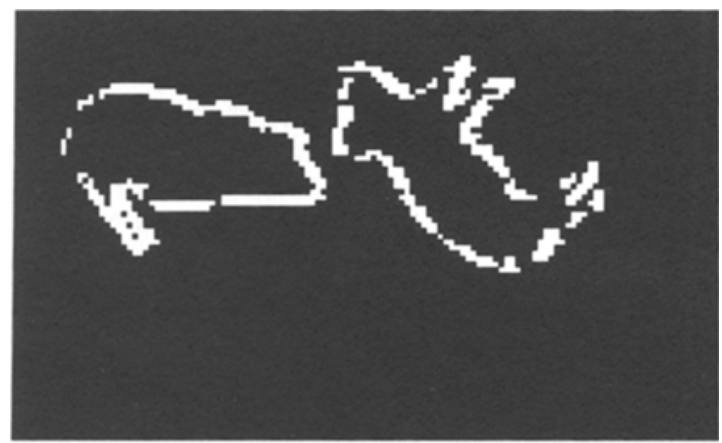

d) shading (threshold $=0.006$ )

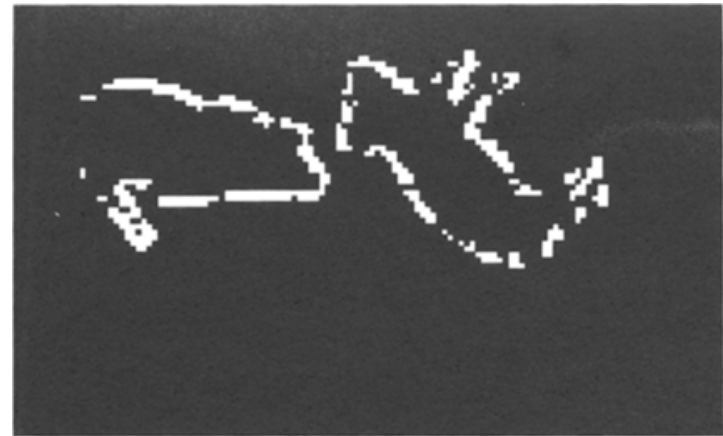

e) shading (threshold $=0.01$ )

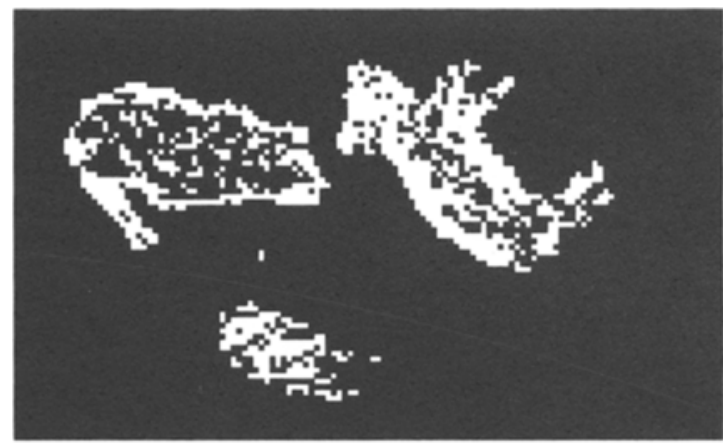

f) likelihood (threshold $=1.8$ )

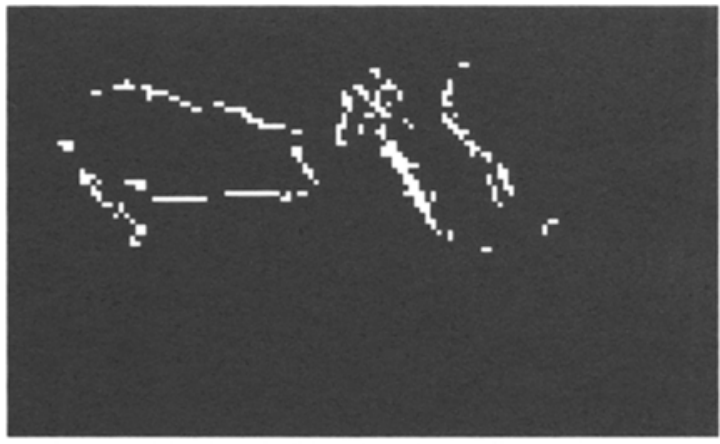

g) likelihood (threshold = 15)

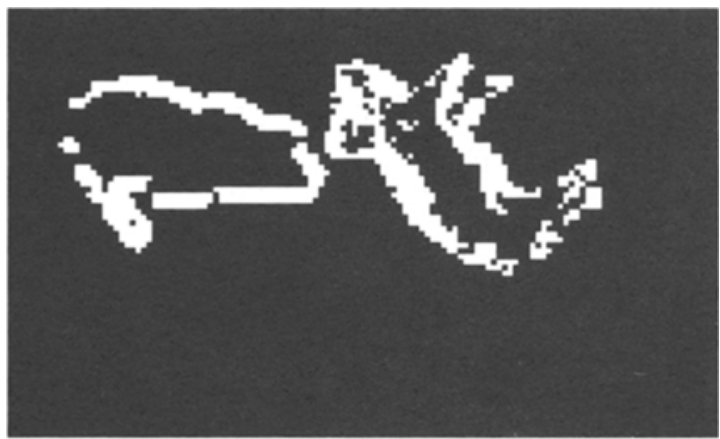

h) result (threshold $=15,0.01$ )

Figure 5. Motion detection of the swine behavior image using a combination of the shading model method and the likelihood ratio method. 
An example of motion detection when illumination changes between images is shown in Figure 4. The first frame was captured under a lower illumination and the second frame under a higher illumination. Two changes or motions were made to the high-illumination image: a slight upward movement of the black circular object and addition of a pen. As can be seen, the likelihood ratio method (Figures $4 \mathrm{e}$ and $4 \mathrm{f}$ ) completely failed to detect these two changes. This failure was attributed to the fact that a single threshold value was insufficient to distinguish the changes from the higher responses caused by the illumination change in other regions, such as the black disk. In comparison, the shading model method (Figures $4 \mathrm{c}$ and $4 \mathrm{~d}$ ) detected the two, thereby showing its total immunity to the illumination variation. The effects of intraframe inspection on reducing detection error are alsc shown in the figure. The intraframe inspection eliminated isolated blocks incorrectly detected at other locations.

Different threshold values also affect the motion detection result. Our experiment showed that, for the likelihood ratio method, the optimal threshold is dependent on the surface properties of the objects. The shading model

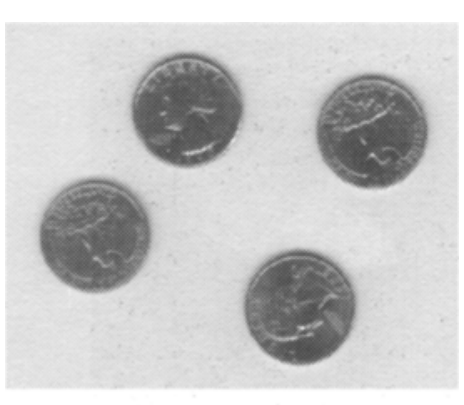

Quarter

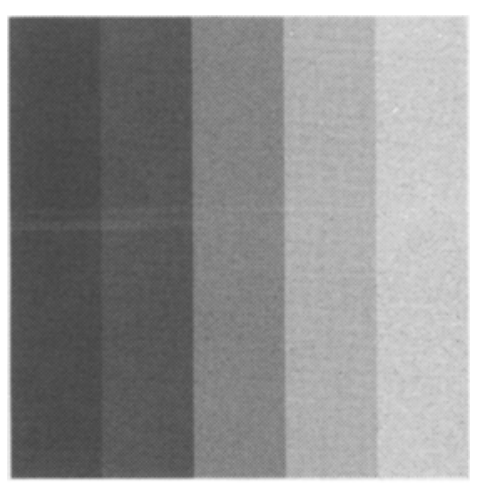

Normal5

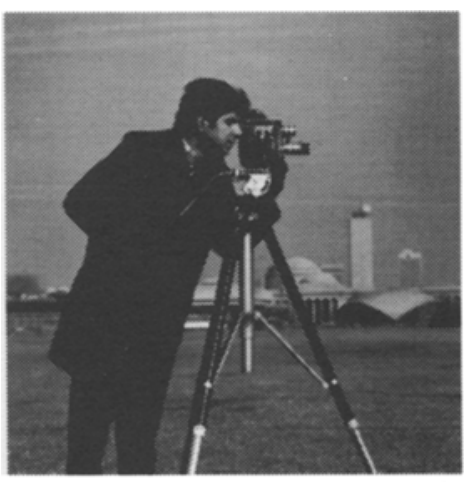

Cameraman
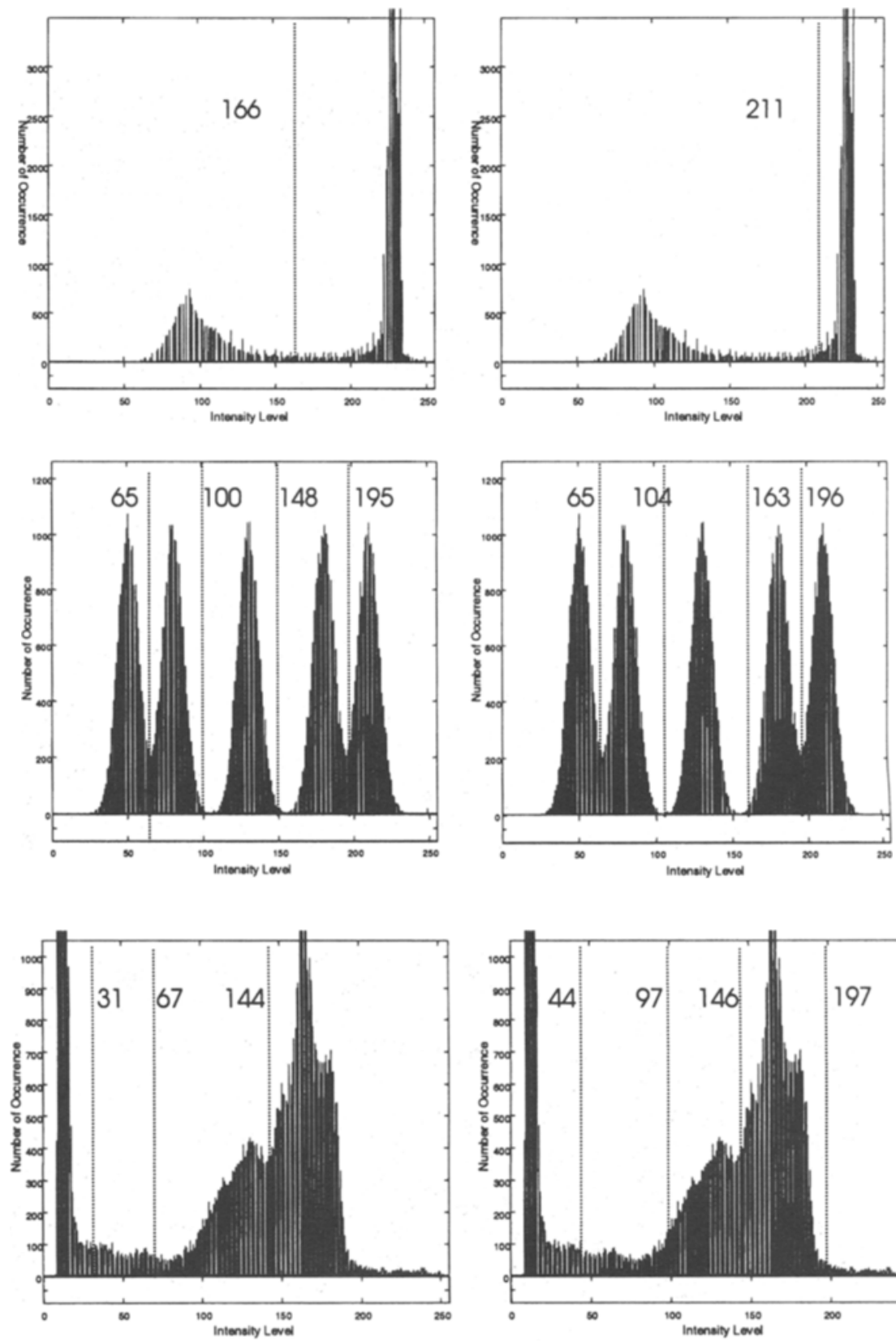

Otsu

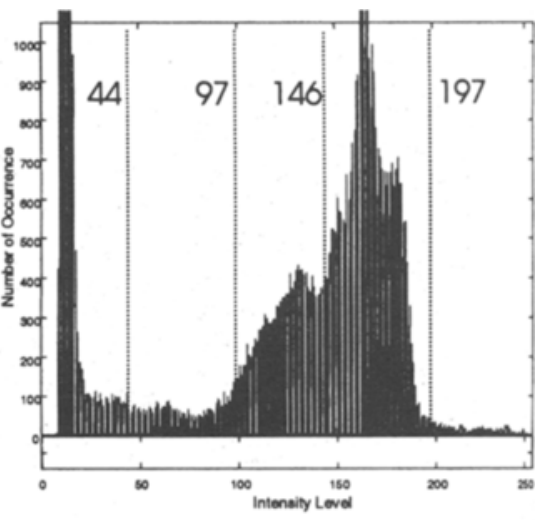

Kapur

Figure 6. Results of multilevel optimal thresholding of selected testing images using Otsu (1979) and Kapur (Kapur et al., 1985) measures. 
Table 1

The Hierarchical Thresholds and Homogeneity Measures of Tested Images

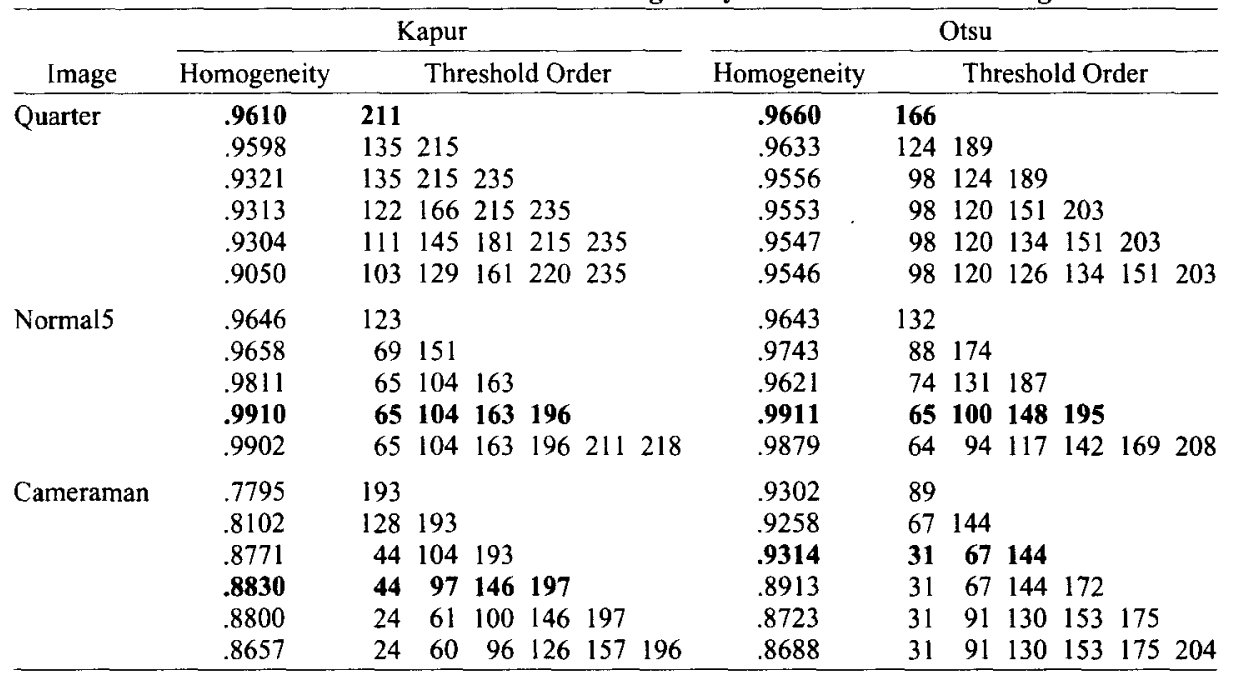

Note-“Kapur" refers to Kapur et al. (1985); "Otsu" refers to Otsu (1979). Values in bold represent the final results.

method can be more sensitive because more regions influenced by a motion can be detected, except for the uncovered or newly covered regions that the likelihood ratio method usually detects. There were situations in which the shading model method failed while the likelihood method succeeded. An example is that a moving object had a small variance and a small mean change relative to that of the background. Under such situations, combining both methods would produce a more reliable result. This is the approach we used for motion detection of the swine behavioral images.

As shown in Figure 5, three swine behavior images were captured to form an image sequence where the top white pig had a right-down movement $(\Delta x=10$ pixels, $\Delta y=-10$ pixels) and the black pig had a left-up movement ( $\Delta x=-10$ pixels, $\Delta y=10$ pixels). The lower white pig did not move. In obtaining the final result, inspection of the motion detection results of the neighboring blocks within the same frame and between two consecutive frames was used. The results from both the likelihood ratio method and the shading model method were combined (i.e., an OR operation). A relatively high threshold (15) was used for the likelihood ratio method and a relatively low threshold $(0.01)$ was used for the shading model method. The result was quite satisfactory.

\section{Image Segmentation}

The performance of the multilevel optimal thresholding method was first evaluated with three unrelated testing images before being applied to the segmentation of the pigs. Figure 6 shows the original image, its histogram, and the multiple thresholds obtained with Otsu (1979) or Kapur (Kapur et al., 1985) optimal measures. The image "Quarter" is a two-class case, and the image "Normal5" is an artificial image of five normal distri- butions with the same variance of 25 and different intensity means of $50,80,130,180$, and 210 . The image "Cameraman" is a multiple-class case whose number of classes is unknown. The final thresholds are indicated on the corresponding histograms. Although the results from these two optimality measures differed somewhat, they both showed the effectiveness of extending the bi-level global thresholding method to multilevel case using the algorithm described above.

Data on the hierarchical thresholds and uniformity measures used to determine the optimal thresholds are shown

\section{Table 2}

The Hierarchical Thresholds and Homogeneity Measures of Swine Postural Images Using the Otsu (1979) Criterion

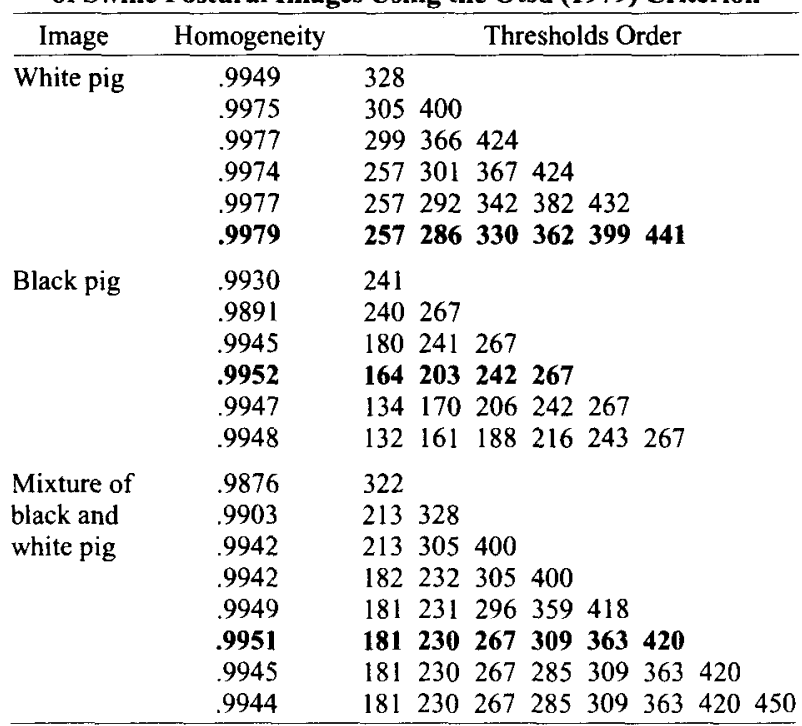

Note--Values in bold represent the final results. 

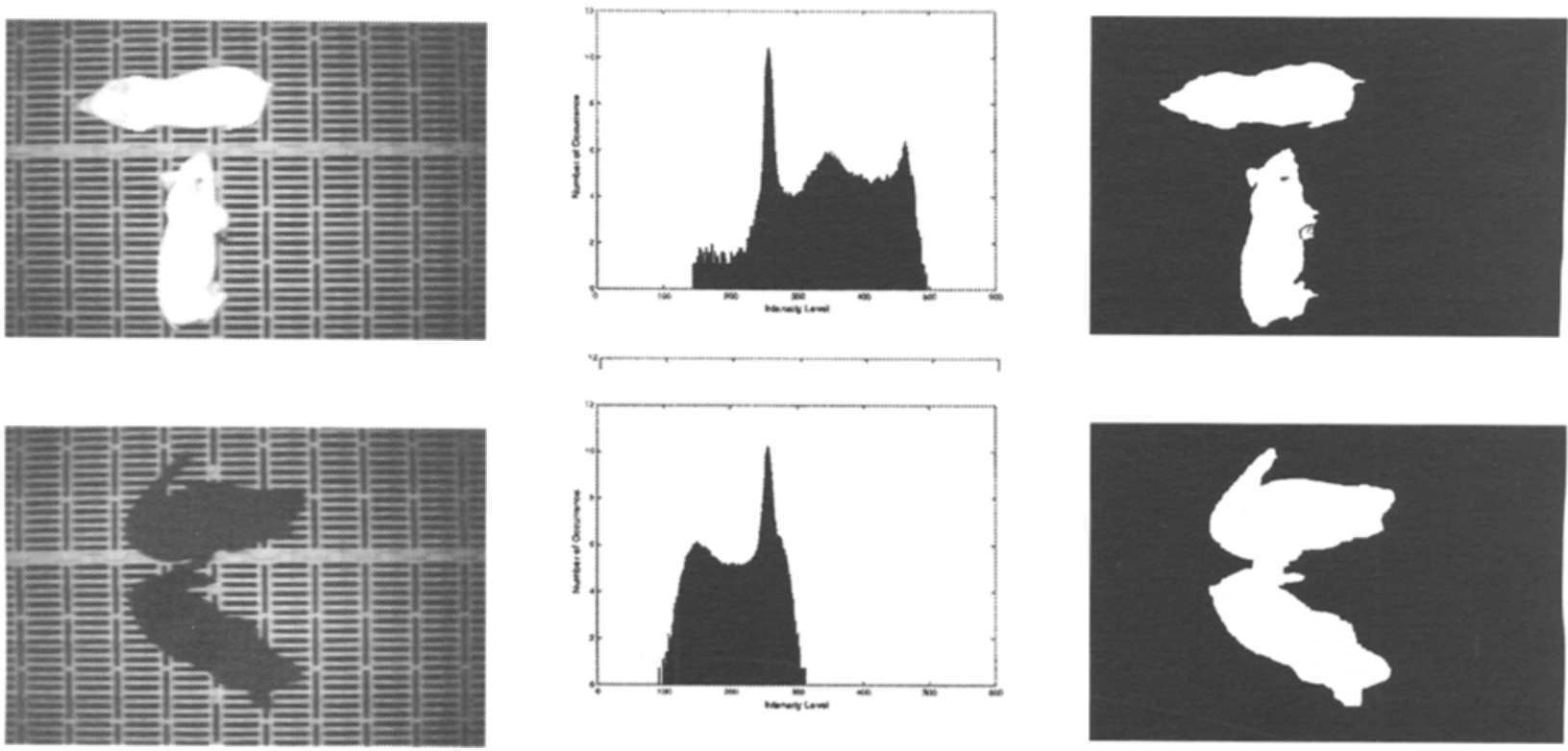

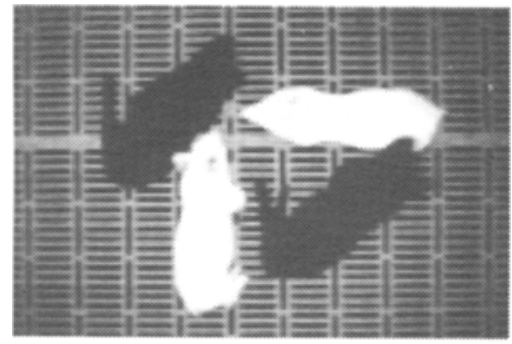

Postural behavior image

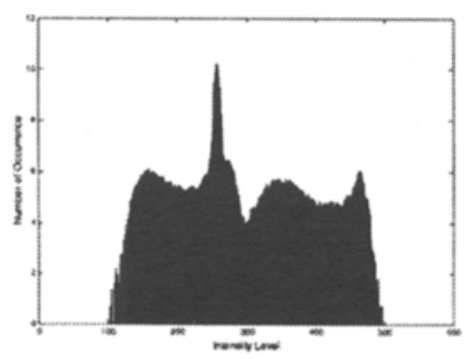

Histogram (in logarithm) and multiple threshold

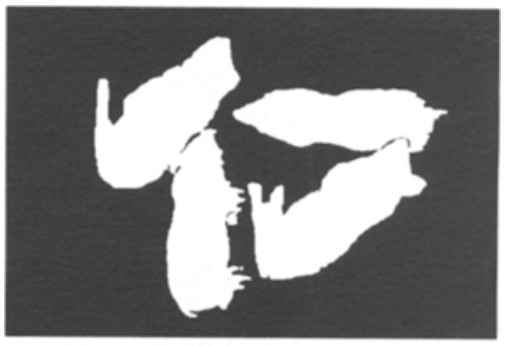

Segmentation result

Figure 7. Segmentation results of swine postural images using multilevel optimal thresholding.

in Table 1. The searching started with bi-level thresholding and eventually reached the optimal level as desired. The bold-faced values in the table represent the final, optimal thresholds that maximize the homogeneity measure.

One problem with the Kapur measure was the multiplepeak feature of its objective function (Kapur et al., 1985) during the search process. Selection among these peaks determines finally whether or not the global maximum can be obtained, and yet no method exists that can guide this procedure. This feature has caused undesirable thresholding results in some multilevel case experiments, although it works well for bi-level cases where choosing a proper threshold among peaks is always possible from the prior knowledge of the application. Thus, for the present study of processing swine postural images, we employed the Otsu measure.

The input of the multilevel optimal thresholding algorithm is the histogram data of the image to be segmented. In this research, the histogram of the extended difference image, instead of the absolute difference image, was used. In extended difference image, the intensity range was ex- tended to $512(-256$ to 255$)$ so that positive and negative intensity differences from different uniform regions could be separated. Compared with the histogram of the absolute difference image, the histogram of the extended difference image has the advantages that the differences between the background pixels are around zero with a narrower range and there are more homogeneous regions. It is thus more meaningful to apply multilevel optimal thresholding.

Segmentation results of the postural images of the pigs in single (white or black) and mixed colors are shown in Figure 7. The final results are the images after they have been processed with morphological filtering operation. The hierarchical process for selection of the thresholds is shown in Table 2, with the final thresholds indicated in bold. A step-by-step illustration of segmentation for each pair of thresholds for the image of mixed pigs is given in Figure 8.

\section{Reference Image Updating}

The effects of different cost structures for the Bayes hypothesis testing on determination of the final exposed 


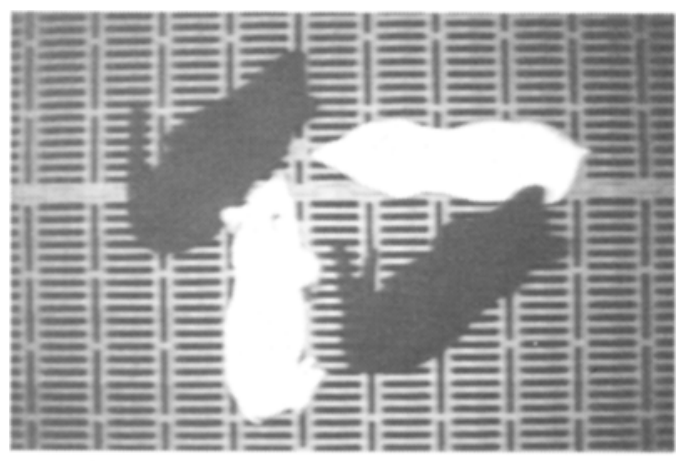

a) original image
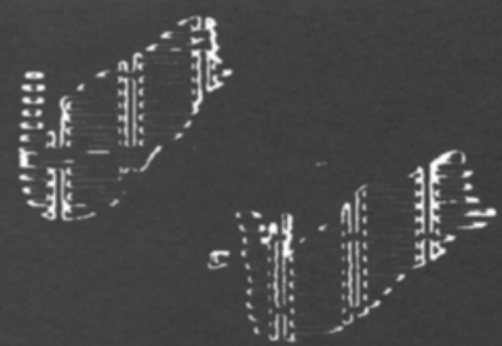

c) Intensity range $(181,230)$

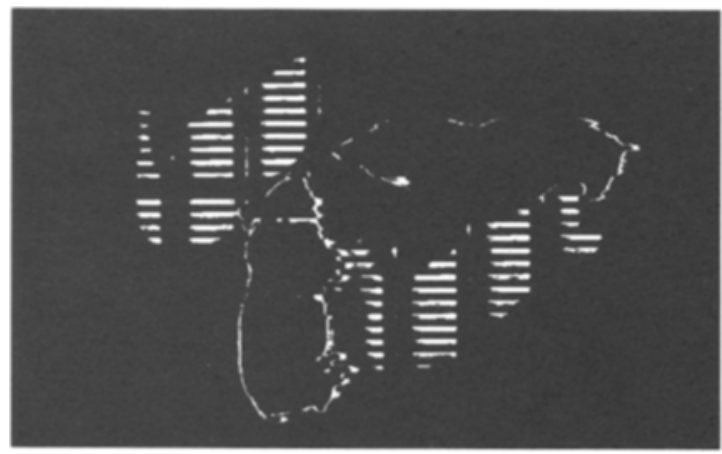

e) Intensity range $(276,309)$

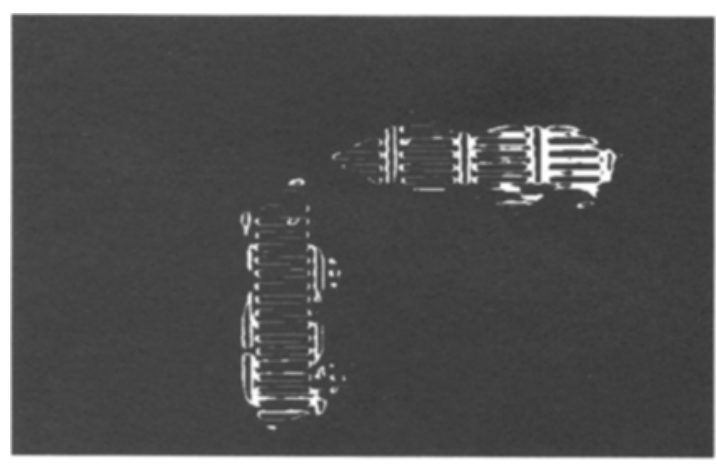

g) Intensity range $(363,420)$
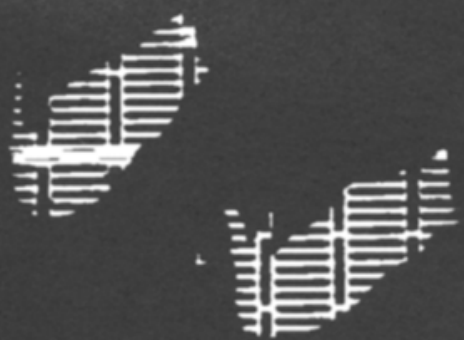

b) Intensity range $(0,181)$

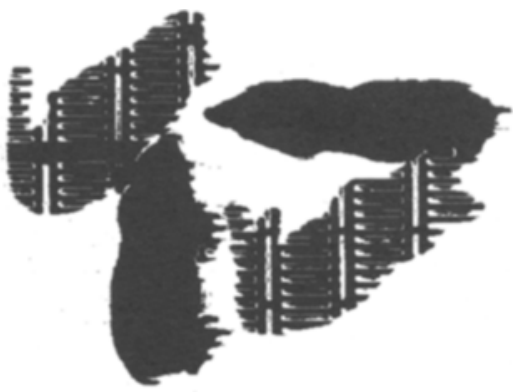

d) Intensity range $(230,276)$

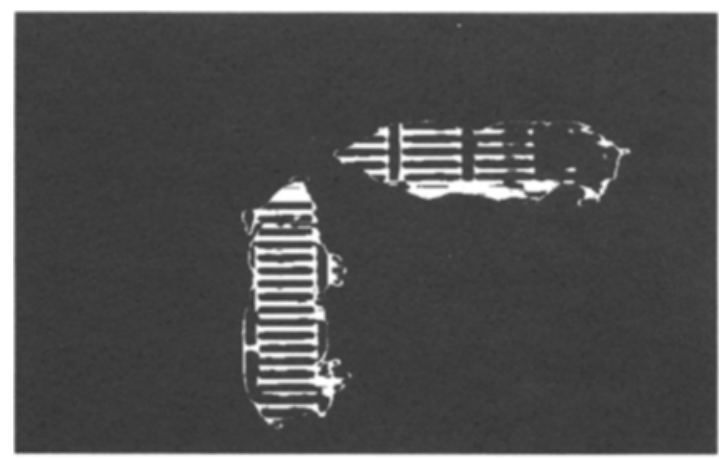

f) Intensity range $(309,363)$

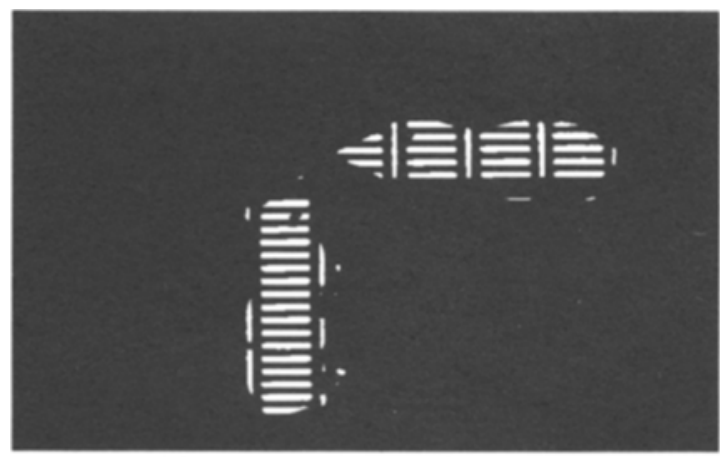

h) Intensity range $(420,511)$

Figure 8. Intermediate, multilevel thresholding results for a mixture of black and white pigs. 


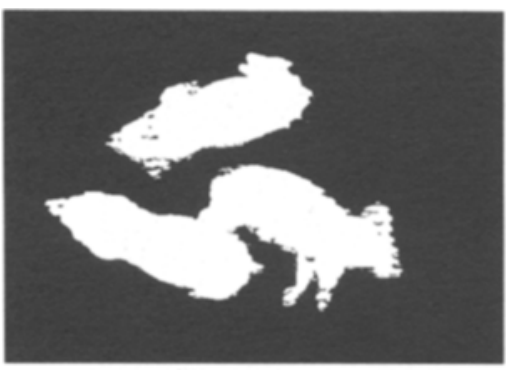

$$
\begin{gathered}
\text { Cost: } C_{b \rightarrow w p}=.8 ; C_{b \rightarrow b p}=.8 \\
C_{w p \rightarrow b}=.2 ; C_{b p \rightarrow b}=.2
\end{gathered}
$$

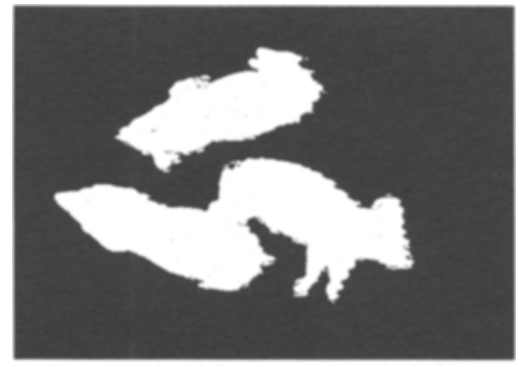

$$
\begin{gathered}
\text { Cost: } C_{b \rightarrow w p}=1 ; C_{b \rightarrow b p}=1 \\
C_{w p \rightarrow b}=1 ; C_{b p \rightarrow b}=1
\end{gathered}
$$

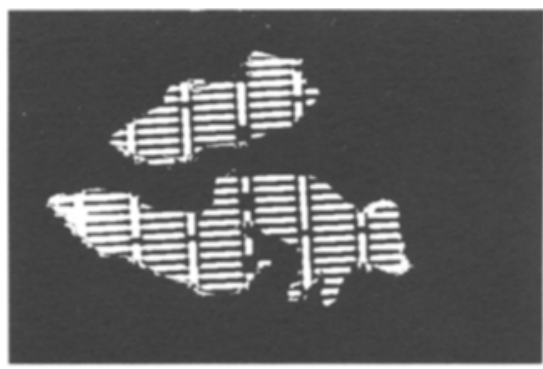

$$
\begin{gathered}
\text { Cost: } C_{b \rightarrow w p}=.2 ; C_{b \rightarrow b p}=.2 \\
C_{w p \rightarrow b}=.8 ; C_{b p \rightarrow b}=.8
\end{gathered}
$$

Figure 9. Effect of different cost structures for background update.

background regions are illustrated in Figure 9. Pixels classified as pigs and background are shown in white and black, respectively. These results were obtained by using the normal distribution assumption. Selection of cost structure should be based on the objective of the application with the largest cost being usually assigned to the most unwanted error. In the current application, the exposed background pixels need to be identified as completely as possible, while the background pixels influenced by the presence of the pigs should be excluded as much as possible. The experimental results were satisfactory with the selected cost structures, as illustrated in Figure 9. The exposed portion of the reference image appearing in black in the figure will be replaced by the corresponding part of the newly captured image.

The use of uniform distribution produced much less desirable results than those for the normal distribution under the same cost structure. This outcome was not surprising since the application-specific information (e.g., mean and variance of the intensity) must be determined and used to set up the statistical models of normal distribution, which therefore describes the application more accurately than the uniform distribution. Nevertheless, because of its computational simplicity, uniform distribution is still a usable method when no other assumptions, such as normal distribution, can be established.

\section{Conclusions}

Computational algorithms for automatic selection and segmentation of swine resting behavior images were developed and evaluated in this study:

1. Combination of the likelihood ratio method and the shading model method along with neighborhood inspection was effective in motion detection for the swine behavior image sequences.

2. Image segmentation was implemented by applying multilevel optimal thresholding to the extended histogram of the difference image between the animal-laden image and the background reference image. The method featured an automatic determination of the number of multilevel thresholds.
3. Background reference image was updated using statistical hypothesis testing that classifies pixels into two or more classes and thus produces the exposed background regions. This background updating produces more homogeneous regions on the animal-laden image, making it more effective to apply the multilevel thresholding technique.

These algorithms will serve the fundamental needs of automatic image segmentation and selection of swine postural behavior used in a novel, behavior-based assessment and control of thermal comfort of the animals.

\section{REFERENCES}

Boon, C. R. (1981). The effect of departure from lower critical temperature on group postural behavior of pigs. Animal Production, 33, 71-79.

Geers, R., Goedseels, M., \& Parduyus, G. (1986). Group postural behavior of growing pigs in relation to air velocity, air and floor temperature. Applied Animal Behaviour Science, 16, 353-362.

Geers, R., Ville, H., Goedseels, V., Houkes, M., Goossens, K., ParDUYNS, G., \& VAN BAEL, J. (1991). Environmental temperature control by the pigs' comfort behavior through image analysis. Transactions of the ASAE, 34, 2583-2586.

$\mathrm{Hu}, \mathrm{J} .(1998)$. Development of image processing algorithms for automatic segmentation and selection of swine postural behaviors used in an interactive environmental control. Unpublished master's thesis, Iowa State University.

Kapur, J. N., Sahoo, P. K., \& Wong, A. K. C. (1985). A new method for gray level picture thresholding using the entropy of histogram. Computer Vision, Graphics, \& Image Processing, 29, 273-285.

Lee, S. U., ChUng, S. Y., \& PARK, R. H. (1990). A comparative performance study of several global thresholding techniques for segmentation. Computer Vision, Graphics, \& Image Processing, 52, 171-190.

LeVINE, M. D., \& NAZIF, A. M. (1985). Dynamic measurement of computer generated image segmentation. IEEE Transactions on Pattern Analysis \& Machine Intelligence, 7, 155-164.

OTsu, N. (1979). A threshold selection method from gray level histograms. IEEE Transactions on Systems, Man \& Cybernetics, 9, 62-66.

PAL, N. R., \& PAL, S. K. (1993). Review on image segmentation techniques. Pattern Recognition, 26, 1277-1294.

Sahoo, P. K., Soltani, S., Wong, A. K. C., \& Chen, Y. C. (1988). A survey of thresholding techniques. Computer Vision, Graphics, \& Image Processing, 41, 233-260.

SHAO, J. (1997). Classification of swine thermal comfort behavior by image processing and neural network. Unpublished doctoral dissertation, Iowa State University.

ShAO, J., XIN, H., \& HaRMON, J. D. (1997). Neural network analysis of 
postural behavior of young swine to determine their thermal comfort state. Transactions of the ASAE, 40,755-760.

Shao, J., XIN, H., \& Harmon, J. D. (1998). Comparison of image feature extraction for classification of swine thermal comfort behavior. Computer \& Electronics in Agriculture, 19, 223-232.

SKIFSTAD, K., \& JAIN, R. (1989). Illumination independent change detection for real world image sequences. Computer Vision, Graphics, \& Image Processing, 46, 387-399.

Wouters, P., Geers, R., Parduyns, G., Goossens, K., Truyen, B., Goedseels, V., \& VAN DEer StUYFT, E. (1990). Image analysis parameters as inputs for automatic environmental temperature control in the piglets' houses. Computers \& Electronics in Agriculture, 5, 233-246.
XIN, H., \& SHAO, J. (1997). Application of machine vision to swine environmental control. Proceedings of Advanced Intelligent Mechatronics 1997. Tokyo: Waseda University.

YAKIMOVSKY, Y. (1976). Boundary and object detection in real world images. Journal of the Association for Computing Machinery, 23, 599618.

YIN, P. Y., \& CHEN, L. H. (1997). A fast iterative scheme for multilevel thresholding methods. Signal Processing, 60, 305-313.

(Manuscript received December 18, 1998; revision accepted for publication October 3, 1999.) 